\title{
Difference of Function on Vector Space over $\mathbb{F}^{1}$
}

\author{
Kenichi Arai \\ Tokyo University of Science \\ Chiba, Japan
}

\author{
Ken Wakabayashi \\ Shinshu University \\ Nagano, Japan
}

\author{
Hiroyuki Okazaki \\ Shinshu University \\ Nagano, Japan
}

\begin{abstract}
Summary. In [11, the definitions of forward difference, backward difference, and central difference as difference operations for functions on $\mathbb{R}$ were formalized. However, the definitions of forward difference, backward difference, and central difference for functions on vector spaces over $\mathbb{F}$ have not been formalized. In cryptology, these definitions are very important in evaluating the security of cryptographic systems [3], 10. Differential cryptanalysis [4] that undertakes a general purpose attack against block ciphers 13 . can be formalized using these definitions. In this article, we formalize the definitions of forward difference, backward difference, and central difference for functions on vector spaces over $\mathbb{F}$. Moreover, we formalize some facts about these definitions.
\end{abstract}

MSC: 39A70 15A03 03B35

Keywords: Mizar formalization; difference of function on vector space over $\mathbb{F}$ MML identifier: VSDIFF_1, version: $8.1 .03 \quad 5.25 .1220$

The notation and terminology used in this paper have been introduced in the following articles: [12, [15], [5], 6], [16], 1], 2], [7], [19], 20], [17], [14, 18], [9], [21], and [8].

From now on $C$ denotes a non empty set, $G_{1}$ denotes a field, $V$ denotes a vector space over $G_{1}, v, u$ denote elements of $V, W$ denotes a subset of $V$, and $f, f_{1}, f_{2}, f_{3}$ denote partial functions from $C$ to $V$.

\footnotetext{
${ }^{1}$ This work was supported by JSPS KAKENHI Grant Number 26730067.
} 
Let us consider $C, G_{1}$, and $V$. Let $f$ be a partial function from $C$ to $V$ and $r$ be an element of $G_{1}$. The functor $r \cdot f$ yielding a partial function from $C$ to $V$ is defined by

(Def. 1) $\operatorname{dom} i t=\operatorname{dom} f$ and for every element $c$ of $C$ such that $c \in \operatorname{dom} i t$ holds $i t_{c}=r \cdot f_{c}$.

Let $f$ be a function from $C$ into $V$. One can check that $r \cdot f$ is total.

Let us consider $v$ and $W$. The functor $v \oplus W$ yielding a subset of $V$ is defined by the term

(Def. 2) $\{v+u: u \in W\}$.

Let $F, G$ be fields, $V$ be a vector space over $F, W$ be a vector space over $G$, $f$ be a partial function from $V$ to $W$, and $h$ be an element of $V$. The functor $\operatorname{Shift}(f, h)$ yielding a partial function from $V$ to $W$ is defined by

(Def. 3) $\operatorname{dom} i t=-h \oplus \operatorname{dom} f$ and for every element $x$ of $V$ such that $x \in$ $-h \oplus \operatorname{dom} f$ holds it $(x)=f(x+h)$.

Now we state the proposition:

(1) Let us consider an element $x$ of $V$ and a subset $A$ of $V$. If $A=$ the carrier of $V$, then $x \oplus A=A$.

Proof: For every object $y, y \in x \oplus A$ iff $y \in A$ by [17, (29), (15), (13)].

Let $F, G$ be fields, $V$ be a vector space over $F, W$ be a vector space over $G$, $f$ be a function from $V$ into $W$, and $h$ be an element of $V$. One can verify that the functor $\operatorname{Shift}(f, h)$ yields a function from $V$ into $W$ and is defined by

(Def. 4) for every element $x$ of $V$, it $(x)=f(x+h)$.

Let $f$ be a partial function from $V$ to $W$. The functor $\Delta_{h}[f]$ yielding a partial function from $V$ to $W$ is defined by the term

(Def. 5) $\operatorname{Shift}(f, h)-f$.

Let $f$ be a function from $V$ into $W$. Observe that $\Delta_{h}[f]$ is quasi total.

Let $f$ be a partial function from $V$ to $W$. The functor $\nabla_{h}[f]$ yielding a partial function from $V$ to $W$ is defined by the term

(Def. 6) $f-\operatorname{Shift}(f,-h)$.

Let $f$ be a function from $V$ into $W$. Let us note that $\nabla_{h}[f]$ is quasi total.

Let $f$ be a partial function from $V$ to $W$. The functor $\delta_{h}[f]$ yielding a partial function from $V$ to $W$ is defined by the term

(Def. 7) $\operatorname{Shift}\left(f,\left(2 \cdot 1_{F}\right)^{-1} \cdot h\right)-\operatorname{Shift}\left(f,-\left(2 \cdot 1_{F}\right)^{-1} \cdot h\right)$.

Let $f$ be a function from $V$ into $W$. One can check that $\delta_{h}[f]$ is quasi total.

The forward difference of $f$ and $h$ yielding a sequence of partial functions from the carrier of $V$ into the carrier of $W$ is defined by

(Def. 8) it $(0)=f$ and for every natural number $n$, it $(n+1)=\Delta_{h}[i t(n)]$. 
We introduce $\vec{\Delta}_{h}[f]$ as a synonym of the forward difference of $f$ and $h$.

From now on $F, G$ denote fields, $V$ denotes a vector space over $F, W$ denotes a vector space over $G, f, f_{1}, f_{2}$ denote functions from $V$ into $W, x, h$ denote elements of $V$, and $r, r_{1}, r_{2}$ denote elements of $G$.

Now we state the propositions:

(2) Let us consider a partial function $f$ from $V$ to $W$. If $x, x+h \in \operatorname{dom} f$, then $\left(\Delta_{h}[f]\right)_{x}=f_{x+h}-f_{x}$.

(3) Let us consider a natural number $n$. Then $\left(\vec{\Delta}_{h}[f]\right)(n)$ is a function from $V$ into $W$.

Proof: Define $\mathcal{X}$ [natural number $] \equiv\left(\vec{\Delta}_{h}[f]\right)\left(\$_{1}\right)$ is a function from $V$ into $W$. For every natural number $k$ such that $\mathcal{X}[k]$ holds $\mathcal{X}[k+1]$. For every natural number $n, \mathcal{X}[n]$ from [1, Sch. 2].

(4) $\left(\Delta_{h}[f]\right)_{x}=f_{x+h}-f_{x}$. The theorem is a consequence of (2).

(5) $\left(\nabla_{h}[f]\right)_{x}=f_{x}-f_{x-h}$.

(6) $\left(\delta_{h}[f]\right)_{x}=f_{x+\left(2 \cdot 1_{F}\right)^{-1} \cdot h}-f_{x-\left(2 \cdot 1_{F}\right)^{-1} \cdot h}$.

From now on $n, m, k$ denote natural numbers.

Now we state the propositions:

(7) If $f$ is constant, then for every $x,\left(\vec{\Delta}_{h}[f]\right)(n+1)_{x}=0_{W}$.

Proof: For every $x, f_{x+h}-f_{x}=0_{W}$ by [17, (15)]. For every $x,\left(\vec{\Delta}_{h}[f]\right)(n+$ $1)_{x}=0_{W}$ by $(3),(4),[17,(15)]$.

(8) $\left(\vec{\Delta}_{h}[r \cdot f]\right)(n+1)_{x}=r \cdot\left(\vec{\Delta}_{h}[f]\right)(n+1)_{x}$.

Proof: Define $\mathcal{X}$ [natural number] $\equiv$ for every $x,\left(\vec{\Delta}_{h}[r \cdot f]\right)\left(\$_{1}+1\right)_{x}=$ $r \cdot\left(\vec{\Delta}_{h}[f]\right)\left(\$_{1}+1\right)_{x}$. For every $k$ such that $\mathcal{X}[k]$ holds $\mathcal{X}[k+1]$ by $(3),(4)$, [9, (23)]. $\mathcal{X}[0]$ by (4), [9, (23)]. For every $n, \mathcal{X}[n]$ from [1, Sch. 2].

(9) $\left(\vec{\Delta}_{h}\left[f_{1}+f_{2}\right]\right)(n+1)_{x}=\left(\vec{\Delta}_{h}\left[f_{1}\right]\right)(n+1)_{x}+\left(\vec{\Delta}_{h}\left[f_{2}\right]\right)(n+1)_{x}$.

Proof: Define $\mathcal{X}$ [natural number $] \equiv$ for every $x,\left(\vec{\Delta}_{h}\left[f_{1}+f_{2}\right]\right)\left(\$_{1}+1\right)_{x}=$ $\left(\vec{\Delta}_{h}\left[f_{1}\right]\right)\left(\$_{1}+1\right)_{x}+\left(\vec{\Delta}_{h}\left[f_{2}\right]\right)\left(\$_{1}+1\right)_{x}$. For every $k$ such that $\mathcal{X}[k]$ holds $\mathcal{X}[k+1]$ by $(3),(4),[17,(27),(28)] . \mathcal{X}[0]$ by $(4)$, [17, $(27),(28)]$. For every $n, \mathcal{X}[n]$ from [1, Sch. 2].

(10) $\left(\vec{\Delta}_{h}\left[f_{1}-f_{2}\right]\right)(n+1)_{x}=\left(\vec{\Delta}_{h}\left[f_{1}\right]\right)(n+1)_{x}-\left(\vec{\Delta}_{h}\left[f_{2}\right]\right)(n+1)_{x}$.

Proof: Define $\mathcal{X}$ [natural number $] \equiv$ for every $x,\left(\vec{\Delta}_{h}\left[f_{1}-f_{2}\right]\right)\left(\$_{1}+1\right)_{x}=$ $\left(\vec{\Delta}_{h}\left[f_{1}\right]\right)\left(\$_{1}+1\right)_{x}-\left(\vec{\Delta}_{h}\left[f_{2}\right]\right)\left(\$_{1}+1\right)_{x} . \mathcal{X}[0]$ by (4), [17, (29), (27)]. For every $k$ such that $\mathcal{X}[k]$ holds $\mathcal{X}[k+1]$ by $(3),(4)$, [17, (29)]. For every $n, \mathcal{X}[n]$ from [1, Sch. 2].

(11) $\left(\vec{\Delta}_{h}\left[r_{1} \cdot f_{1}+r_{2} \cdot f_{2}\right]\right)(n+1)_{x}=r_{1} \cdot\left(\vec{\Delta}_{h}\left[f_{1}\right]\right)(n+1)_{x}+r_{2} \cdot\left(\vec{\Delta}_{h}\left[f_{2}\right]\right)(n+1)_{x}$. The theorem is a consequence of $(3),(9)$, and (8).

(12) $\left(\vec{\Delta}_{h}[f]\right)(1)_{x}=(\operatorname{Shift}(f, h))_{x}-f_{x}$. The theorem is a consequence of $(4)$. 
Let $F, G$ be fields, $V$ be a vector space over $F, h$ be an element of $V, W$ be a vector space over $G$, and $f$ be a function from $V$ into $W$. The backward difference of $f$ and $h$ yielding a sequence of partial functions from the carrier of $V$ into the carrier of $W$ is defined by

(Def. 9) $\quad i t(0)=f$ and for every natural number $n$, it $(n+1)=\nabla_{h}[i t(n)]$.

The backward difference of $f$ and $h$ yielding a sequence of partial functions from the carrier of $V$ into the carrier of $W$ is defined by

(Def. 10) $i t(0)=f$ and for every natural number $n$, it $(n+1)=\nabla_{h}[i t(n)]$.

We introduce $\vec{\nabla}_{h}[f]$ as a synonym of the backward difference of $f$ and $h$.

Now we state the propositions:

(13) Let us consider a natural number $n$. Then $\left(\vec{\nabla}_{h}[f]\right)(n)$ is a function from $V$ into $W$.

Proof: Define $\mathcal{X}$ [natural number $] \equiv\left(\vec{\nabla}_{h}[f]\right)\left(\$_{1}\right)$ is a function from $V$ into $W$. For every natural number $k$ such that $\mathcal{X}[k]$ holds $\mathcal{X}[k+1]$. For every natural number $n, \mathcal{X}[n]$ from [1, Sch. 2].

(14) If $f$ is constant, then for every $x,\left(\vec{\nabla}_{h}[f]\right)(n+1)_{x}=0_{W}$.

Proof: For every $x, f_{x}-f_{x-h}=0_{W}$ by [17, (15)]. For every $x,\left(\vec{\nabla}_{h}[f]\right)(n+$ $1)_{x}=0_{W}$ by (13), (5), [17, (15)].

(15) $\left(\vec{\nabla}_{h}[r \cdot f]\right)(n+1)_{x}=r \cdot\left(\vec{\nabla}_{h}[f]\right)(n+1)_{x}$.

Proof: Define $\mathcal{X}$ [natural number $] \equiv$ for every $x,\left(\vec{\nabla}_{h}[r \cdot f]\right)\left(\$_{1}+1\right)_{x}=$ $r \cdot\left(\vec{\nabla}_{h}[f]\right)\left(\$_{1}+1\right)_{x}$. For every $k$ such that $\mathcal{X}[k]$ holds $\mathcal{X}[k+1]$ by $(13),(5)$, [9, (23)]. $\mathcal{X}[0]$ by (5), [9, (23)]. For every $n, \mathcal{X}[n]$ from [1, Sch. 2].

(16) $\left(\vec{\nabla}_{h}\left[f_{1}+f_{2}\right]\right)(n+1)_{x}=\left(\vec{\nabla}_{h}\left[f_{1}\right]\right)(n+1)_{x}+\left(\vec{\nabla}_{h}\left[f_{2}\right]\right)(n+1)_{x}$.

Proof: Define $\mathcal{X}$ [natural number $] \equiv$ for every $x,\left(\vec{\nabla}_{h}\left[f_{1}+f_{2}\right]\right)\left(\$_{1}+1\right)_{x}=$ $\left(\vec{\nabla}_{h}\left[f_{1}\right]\right)\left(\$_{1}+1\right)_{x}+\left(\vec{\nabla}_{h}\left[f_{2}\right]\right)\left(\$_{1}+1\right)_{x}$. For every $k$ such that $\mathcal{X}[k]$ holds $\mathcal{X}[k+1]$ by $(13),(5),[17,(27),(28)] . \mathcal{X}[0]$ by (5), [17, (27), (28)]. For every $n, \mathcal{X}[n]$ from [1, Sch. 2].

$$
\left(\vec{\nabla}_{h}\left[f_{1}-f_{2}\right]\right)(n+1)_{x}=\left(\vec{\nabla}_{h}\left[f_{1}\right]\right)(n+1)_{x}-\left(\vec{\nabla}_{h}\left[f_{2}\right]\right)(n+1)_{x} .
$$

Proof: Define $\mathcal{X}$ [natural number $] \equiv$ for every $x,\left(\vec{\nabla}_{h}\left[f_{1}-f_{2}\right]\right)\left(\$_{1}+1\right)_{x}=$ $\left(\vec{\nabla}_{h}\left[f_{1}\right]\right)\left(\$_{1}+1\right)_{x}-\left(\vec{\nabla}_{h}\left[f_{2}\right]\right)\left(\$_{1}+1\right)_{x} . \mathcal{X}[0]$ by $(5)$, [17, $\left.(29),(27)\right]$. For every $k$ such that $\mathcal{X}[k]$ holds $\mathcal{X}[k+1]$ by $(13),(5)$, [17, (29), (27)]. For every $n$, $\mathcal{X}[n]$ from [1, Sch. 2].

$$
\left(\vec{\nabla}_{h}\left[r_{1} \cdot f_{1}+r_{2} \cdot f_{2}\right]\right)(n+1)_{x}=r_{1} \cdot\left(\vec{\nabla}_{h}\left[f_{1}\right]\right)(n+1)_{x}+r_{2} \cdot\left(\vec{\nabla}_{h}\left[f_{2}\right]\right)(n+1)_{x}
$$

The theorem is a consequence of (16) and (15).

(19) $\left(\vec{\nabla}_{h}[f]\right)(1)_{x}=f_{x}-(\operatorname{Shift}(f,-h))_{x}$. The theorem is a consequence of $(5)$.

Let $F, G$ be fields, $V$ be a vector space over $F, h$ be an element of $V, W$ be a vector space over $G$, and $f$ be a partial function from $V$ to $W$. The central 
difference of $f$ and $h$ yielding a sequence of partial functions from the carrier of $V$ into the carrier of $W$ is defined by

(Def. 11) $\quad i t(0)=f$ and for every natural number $n$, it $(n+1)=\delta_{h}[i t(n)]$.

We introduce $\vec{\delta}_{h}[f]$ as a synonym of the central difference of $f$ and $h$.

Now we state the propositions:

(20) Let us consider a natural number $n$. Then $\left(\vec{\delta}_{h}[f]\right)(n)$ is a function from $V$ into $W$.

Proof: Define $\mathcal{X}$ [natural number] $\equiv\left(\vec{\delta}_{h}[f]\right)\left(\$_{1}\right)$ is a function from $V$ into $W$. For every natural number $k$ such that $\mathcal{X}[k]$ holds $\mathcal{X}[k+1]$. For every natural number $n, \mathcal{X}[n]$ from [1, Sch. 2].

(21) If $f$ is constant, then for every $x,\left(\vec{\delta}_{h}[f]\right)(n+1)_{x}=0_{W}$.

Proof: Define $\mathcal{X}$ [natural number] $\equiv$ for every $x,\left(\vec{\delta}_{h}[f]\right)\left(\$_{1}+1\right)_{x}=0_{W}$. For every $x, f_{x+\left(2 \cdot 1_{F}\right)^{-1} \cdot h}-f_{x-\left(2 \cdot 1_{F}\right)^{-1} \cdot h}=0_{W}$ by [17, (15)]. $\mathcal{X}[0]$. For every $k$ such that $\mathcal{X}[k]$ holds $\mathcal{X}[k+1]$ by $(20),(6),[17,(13)]$. For every $n, \mathcal{X}[n]$ from [1, Sch. 2].

(22) $\left(\vec{\delta}_{h}[r \cdot f]\right)(n+1)_{x}=r \cdot\left(\vec{\delta}_{h}[f]\right)(n+1)_{x}$.

Proof: Define $\mathcal{X}$ [natural number] $\equiv$ for every $x,\left(\vec{\delta}_{h}[r \cdot f]\right)\left(\$_{1}+1\right)_{x}=$ $r \cdot\left(\vec{\delta}_{h}[f]\right)\left(\$_{1}+1\right)_{x}$. For every $k$ such that $\mathcal{X}[k]$ holds $\mathcal{X}[k+1]$ by $(20),(6)$, [9, (23)]. $\mathcal{X}[0]$ by (6), [9, (23)]. For every $n, \mathcal{X}[n]$ from [1, Sch. 2].

(23) $\left(\vec{\delta}_{h}\left[f_{1}+f_{2}\right]\right)(n+1)_{x}=\left(\vec{\delta}_{h}\left[f_{1}\right]\right)(n+1)_{x}+\left(\vec{\delta}_{h}\left[f_{2}\right]\right)(n+1)_{x}$.

Proof: Define $\mathcal{X}$ [natural number $] \equiv$ for every $x,\left(\vec{\delta}_{h}\left[f_{1}+f_{2}\right]\right)\left(\$_{1}+1\right)_{x}=$ $\left(\vec{\delta}_{h}\left[f_{1}\right]\right)\left(\$_{1}+1\right)_{x}+\left(\vec{\delta}_{h}\left[f_{2}\right]\right)\left(\$_{1}+1\right)_{x}$. For every $k$ such that $\mathcal{X}[k]$ holds $\mathcal{X}[k+1]$ by $(20),(6),[17,(27),(28)] . \mathcal{X}[0]$ by $(6),[17,(27),(28)]$. For every $n, \mathcal{X}[n]$ from [1, Sch. 2].

(24) $\left(\vec{\delta}_{h}\left[f_{1}-f_{2}\right]\right)(n+1)_{x}=\left(\vec{\delta}_{h}\left[f_{1}\right]\right)(n+1)_{x}-\left(\vec{\delta}_{h}\left[f_{2}\right]\right)(n+1)_{x}$.

ProOF: Define $\mathcal{X}$ [natural number $] \equiv$ for every $x,\left(\vec{\delta}_{h}\left[f_{1}-f_{2}\right]\right)\left(\$_{1}+1\right)_{x}=$ $\left(\vec{\delta}_{h}\left[f_{1}\right]\right)\left(\$_{1}+1\right)_{x}-\left(\vec{\delta}_{h}\left[f_{2}\right]\right)\left(\$_{1}+1\right)_{x} . \mathcal{X}[0]$ by $(6)$, [17, $\left.(29),(27),(28)\right]$. For every $k$ such that $\mathcal{X}[k]$ holds $\mathcal{X}[k+1]$ by $(20),(6),[17,(29),(27),(28)]$. For every $n, \mathcal{X}[n]$ from [1, Sch. 2].

(25) $\left(\vec{\delta}_{h}\left[r_{1} \cdot f_{1}+r_{2} \cdot f_{2}\right]\right)(n+1)_{x}=r_{1} \cdot\left(\vec{\delta}_{h}\left[f_{1}\right]\right)(n+1)_{x}+r_{2} \cdot\left(\vec{\delta}_{h}\left[f_{2}\right]\right)(n+1)_{x}$. The theorem is a consequence of $(23)$ and $(22)$.

(26) $\left(\vec{\delta}_{h}[f]\right)(1)_{x}=\left(\operatorname{Shift}\left(f,\left(2 \cdot 1_{F}\right)^{-1} \cdot h\right)\right)_{x}-\left(\operatorname{Shift}\left(f,-\left(2 \cdot 1_{F}\right)^{-1} \cdot h\right)\right)_{x}$. The theorem is a consequence of $(6)$.

(27) $\left(\vec{\Delta}_{h}[f]\right)(n)_{x}=\left(\vec{\nabla}_{h}[f]\right)(n)_{x+n \cdot h}$.

Proof: Define $\mathcal{X}$ [natural number] $\equiv$ for every $x,\left(\vec{\Delta}_{h}[f]\right)\left(\$_{1}\right)_{x}$ $=\left(\vec{\nabla}_{h}[f]\right)\left(\$_{1}\right)_{x+\$_{1} \cdot h}$. For every $k$ such that $\mathcal{X}[k]$ holds $\mathcal{X}[k+1]$ by $(3)$, [15, (13), (15)], [17, (4), (15), (28)]. $\mathcal{X}[0]$ by [17, (4)], [15, (12)]. For every $n$, $\mathcal{X}[n]$ from [1, Sch. 2]. 
Let us assume that $1_{F} \neq-1_{F}$. Now we state the propositions:

$$
\left(\vec{\Delta}_{h}[f]\right)(2 \cdot n)_{x}=\left(\vec{\delta}_{h}[f]\right)(2 \cdot n)_{x+n \cdot h} \cdot
$$

Proof: Define $\mathcal{X}[$ natural number $] \equiv$ for every $x,\left(\vec{\Delta}_{h}[f]\right)\left(2 \cdot \$_{1}\right)_{x}=\left(\vec{\delta}_{h}[f]\right)(2$. $\left.\$_{1}\right)_{x+\$_{1} \cdot h}$. For every $k$ such that $\mathcal{X}[k]$ holds $\mathcal{X}[k+1]$ by $[15$, (13), (15)], [17, (27), (28), (15)]. $\mathcal{X}[0]$ by [17, (4)], [15, (12)]. For every $n, \mathcal{X}[n]$ from [1, Sch. 2].

(29) $\left(\vec{\Delta}_{h}[f]\right)(2 \cdot n+1)_{x}=\left(\vec{\delta}_{h}[f]\right)(2 \cdot n+1)_{x+n \cdot h+\left(2 \cdot 1_{F}\right)^{-1} \cdot h}$.

Proof: $2 \cdot 1_{F} \neq 0_{F}$ by $\left[15\right.$, (13), (15)]. $\left(\vec{\delta}_{h}[f]\right)(2 \cdot n)$ is a function from $V$ into $W .\left(\vec{\Delta}_{h}[f]\right)(2 \cdot n)$ is a function from $V$ into $W$.

ACKNowledgement: We sincerely thank Professor Yasunari Shidama for his helpful advices.

\section{REFERENCES}

[1] Grzegorz Bancerek. The fundamental properties of natural numbers Formalized Mathematics, 1(1):41-46, 1990.

[2] Grzegorz Bancerek. The ordinal numbers Formalized Mathematics, 1(1):91-96, 1990.

[3] E. Biham and A. Shamir. Differential cryptanalysis of DES-like cryptosystems. Lecture Notes in Computer Science, 537:2-21, 1991.

[4] E. Biham and A. Shamir. Differential cryptanalysis of the full 16-round DES. Lecture Notes in Computer Science, 740:487-496, 1993.

[5] Czesław Byliński. Functions and their basic properties Formalized Mathematics, 1(1): 55-65, 1990.

[6] Czesław Byliński. Functions from a set to a set Formalized Mathematics, 1(1):153-164, 1990.

[7] Czesław Byliński. Partial functions. Formalized Mathematics, 1(2):357-367, 1990.

[8] Czesław Byliński. Some basic properties of sets Formalized Mathematics, 1(1):47-53, 1990.

[9] Eugeniusz Kusak, Wojciech Leończuk, and Michał Muzalewski. Abelian groups, fields and vector spaces. Formalized Mathematics, 1(2):335-342, 1990.

[10] X. Lai. Higher order derivatives and differential cryptoanalysis. Communications and Cryptography, pages 227-233, 1994.

[11] Bo Li, Yan Zhang, and Xiquan Liang. Difference and difference quotient. Formalized Mathematics, 14(3):115-119, 2006. doi 10.2478/v10037-006-0014-z

[12] Michał Muzalewski and Wojciech Skaba. From loops to Abelian multiplicative groups with zero. Formalized Mathematics, 1(5):833-840, 1990.

[13] Hiroyuki Okazaki and Yasunari Shidama. Formalization of the data encryption standard. Formalized Mathematics, 20(2):125-146, 2012. doi 10.2478/v10037-012-0016-y

[14] Beata Perkowska. Functional sequence from a domain to a domain Formalized Mathematics, 3(1):17-21, 1992.

[15] Christoph Schwarzweller. The binomial theorem for algebraic structures Formalized Mathematics, 9(3):559-564, 2001.

[16] Wojciech A. Trybulec. Groups Formalized Mathematics, 1(5):821-827, 1990.

[17] Wojciech A. Trybulec. Vectors in real linear space. Formalized Mathematics, 1(2):291-296, 1990.

[18] Zinaida Trybulec. Properties of subsets Formalized Mathematics, 1(1):67-71, 1990.

[19] Edmund Woronowicz. Relations and their basic properties. Formalized Mathematics, 1 (1):73-83, 1990. 
[20] Edmund Woronowicz. Relations defined on sets Formalized Mathematics, 1(1):181-186, 1990.

[21] Hiroshi Yamazaki and Yasunari Shidama. Algebra of vector functions Formalized Mathematics, 3(2):171-175, 1992.

Received September 26, 2014 